\title{
Design and Implementation of Inspection System for Lift Based on Android Platform
}

\author{
Yan Zhang ${ }^{1, ~ a ~}$, Yanping $\mathrm{Hu}^{2, b}$ \\ ${ }^{1}$ Dalian University of Technology, China \\ 2 Dalian University of Technology, China \\ azhangyan1225@126.com, bhypok@dlut.edu.cn
}

Keywords: lift inspection system, route plan, greedy algorithms

\begin{abstract}
In order to develop a more convenient way of lift inspection, this paper designs and implements the lift inspection system. This system uses SQLite to store the cache, so that inspectors can record without network. In this System, inspectors sign in in a new way-the QR Code. This paper uses a open source tool named ZXing to accomplish this function. The system also put map--BaiduMap in it so that inspectors can easily see the locations of their sites to be inspected. Also the system uses the greedy algorithms to plan the routes to get the nearest path to get every site. As a result the system has improve the efficiency of inspection.
\end{abstract}

\section{Introduction}

Since the building is becoming higher and higher, lift becomes indispensable in people's life. We can use lift everywhere, from malls to schools, from home to company. Lift needs to be maintained during the period of being used to ensure the safety. So the inspectors must inspect lift regularly. The traditional way to record is by manual recording [1], which is bad for the preserve and the statistics of the record. And fake record and missing record can't be avoided in this way [2]. Also it takes a long time to send the record to the managers. So it becomes very necessary to develop a new way of inspection of lift.

Mobile internet is a new tendency in our life. It takes full advantage of the mobile and internet. So it can be both networked and mobility [3]. With the rapid development of the mobile internet in recent years, the mobile device is becoming more and more popular among the people, including IOS device, Windows phone device and Android device. Among these devices, Android device is the most popular because of its open source, stability and portability. We almost use smart phone and pad every day. Their portability provides us a new way of inspection.

Starting with whole design schemes, this paper designs and implements the lift inspection system. Also this paper introduces the key technologies and the function modules. Finally this paper analyzes the advantages and shortages of the system in order to give some advices to the coming versions.

\section{Whole Schemes Design}

The whole system is composed of three parts: server, web client and android client. The server is used to realize data persistence and deal with the requests come from web client and android client. Web client is used to manage the data such as add inspection tasks to the inspectors, add inspector to the system, add sites to the system and so on. Android client is used by inspectors to do the inspection work, such as get tasks added by managers, record the detail of the lifts, and send the record to the server and so on. The scheme design shows in fig. 1 below.

Server Design. There are two data persistence layer framework can be used when we start to build server. One is Hibernate and the other one is Mybatis. They both are very excellent framework. Hibernate framework can realize entity-relational mapping without writing SQL query statement. Programmer can program in the way of object-oriented. It provides very comprehensive encapsulation of JDBC, which also restrict the flexibility of SQL query. When the query is very 
complicated, the operation will make troubles [4]. So this system uses the Mybatis framework. As for the database, this system uses MySQL because of its powerful functions and speediness.

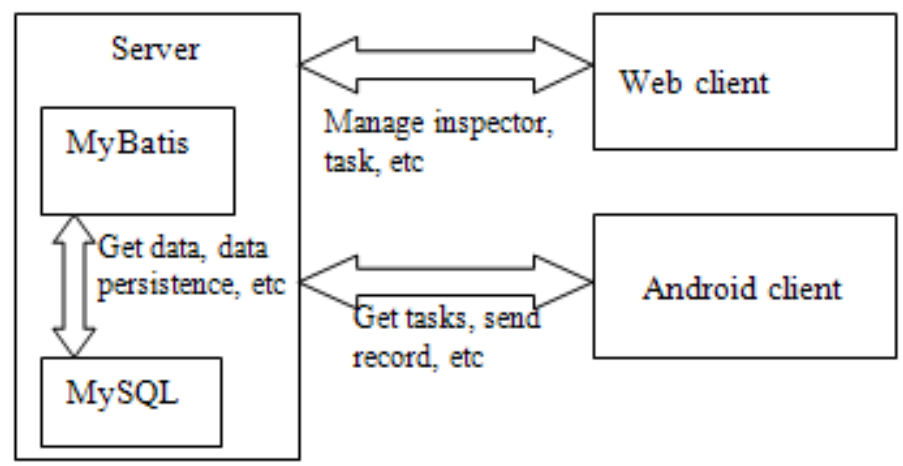

Fig.1 Whole Design Scheme

Web Client Design. This paper uses SpringMVC framework to build web client. MVC(Model-View-Controller) pattern is a Software architecture patterns. It is composed of three parts: model, view and controller [5]. MVC pattern has the features of low coupling, high reusability, fast deployment, high maintainability and so on. SpringMVC has realized the core of MVC. Also SpringMVC provides many functions about MVC for controller and program processing. It can be highly decupled by programming when it adds inverse control to MVC. It also can change component dynamically by simply configuration [6].

Android Client Design. Android client is developed by the language of java in the Eclipse IDE. The Eclipse has android SDK plugged in.

\section{Design of Android Client Function Modules}

This system is mainly used by inspectors for inspection. The main aim of the system is to complete four function modules: task management, route plan, inspection management, setting. The function modules are shown in fig. 2 below.

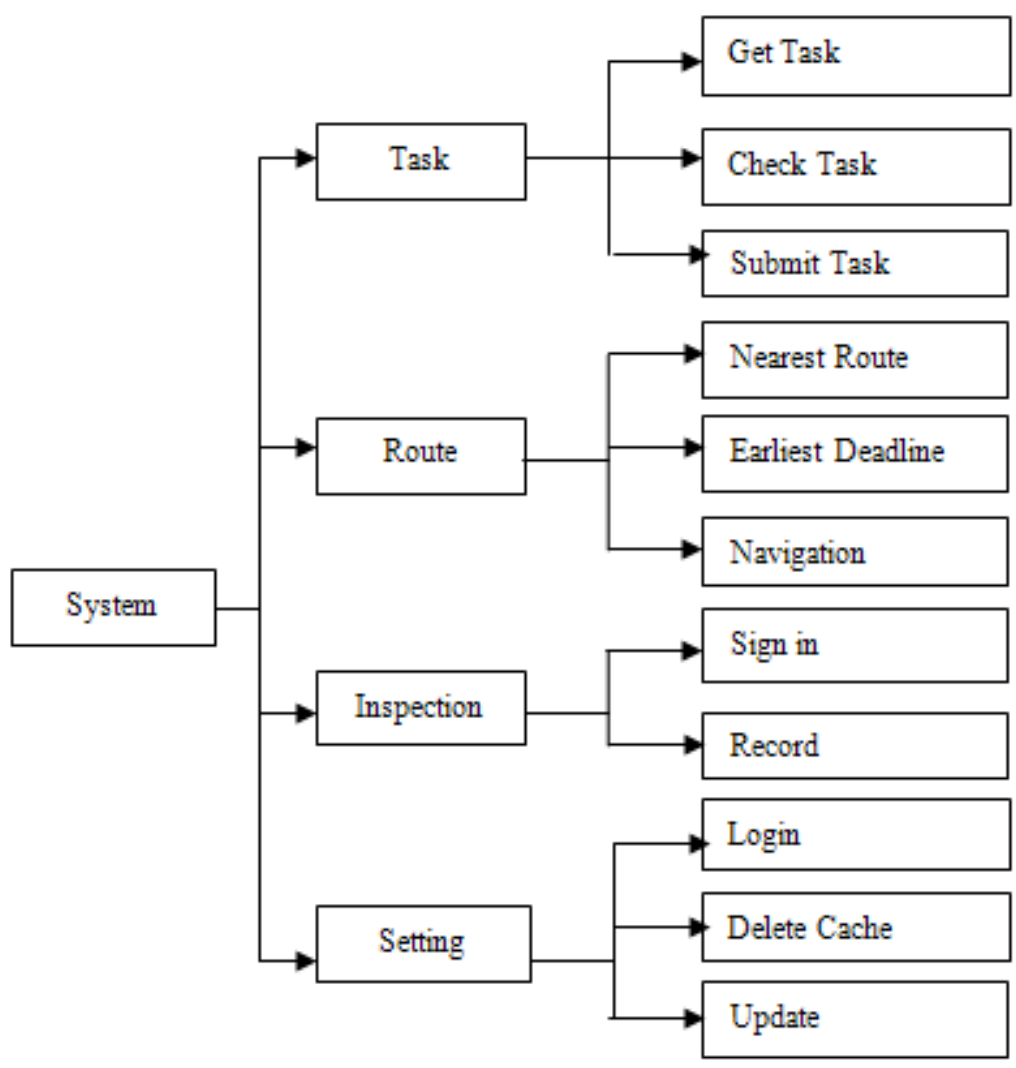

Fig.2 Android Client Function Modules 
Task. The main aim of this module is to manage the tasks. This module is composed of three parts: getting tasks, viewing tasks, and submitting tasks. When there are new tasks are pushed to the terminal, inspectors can download the tasks to the local-storage. And inspectors can view all the tasks to see which one is not completed. After record inspectors can submit the record to the server so that managers can see and analyze the corresponding records.

Route. This android client uses BaiduMap API to show the locations of the sites to be inspected. In this way inspectors can easily find the locations they should reach. Also this paper has the function of planning route. There are two way of planning route: the nearest way and the earliest deadline way. Additionally, inspectors can use navigation to get the ways to the sites easily.

Inspection. This part is the work after inspectors arrived in the inspection sites. This module includes two parts: sign in and record. Sign in refers to signing in in the way of QR Code (abbreviated from Quick Response Code). When inspectors arrive at a site, they need to scan the corresponding QR Code. In this way, they record their position coordinates. The corporation can avoid fake sign in by this way. After sign they should record the detail of the lift. When there is something wrong, they can take a photo or video the problem to store in the local-storage. Finally they submit the record to the server in the task module so that managers can see and analyze the record.

Setting. This part mainly provides some accessory functions to the client. It has three parts: login, delete cache, and version update. The part of login is mainly used to change inspector when there are more than one inspectors using the one terminal. The part of deleting cache is mainly used to delete the submitted record to make room for other data in the terminal. The version update part is mainly used to make sure that the client in the terminal is the latest released.

\section{Implementation of Android Client}

Implementation of data cache. Data cache means store the data, such as task, record, in the local-storage so that inspectors can view the data even without network. This system uses SQLite to cache the data. SQLite database is an open source embedded database written by D.Richard Hipp in C. It can support as much as $2 \mathrm{~T}$ database. All the source code is about 30,000 lines [7]. SQLite has the properties of supporting ACID transactions, no configuration, no installing and configuration of management, little enough, fast running and so on[8]. Android operate system integrates the SQLite database. And also the operation of SQLite can occupy less resources than JDBC connection. So the SQLite is very suitable in the mobile devices. When inspectors record, they store the record data in the local-storage firstly. Then they would submit the record to the server in the task submit of the task module. By this way, they can record the detail even in the environment without network. And they can submit the data when there is network after record.

Sign in with QR Code. QR Code was developed by Denso Wave Inc. in 1994to support object identification and tracking in industrial processes of Toyota [10]. It belongs to the category of 2Dbarcodes, sometimes also referred as matrix codes. QR Code has the properties of high capacity, powerful error-correcting ability, wide range of coding, encryption mechanism can be introduced and so on. The generation and recognition of the QR Code is implemented by the open source tool named ZXing. The generation is completed in the web client and the recognition is completed in the android client. We put the site's ID information to the QR Code when generating the QR Code. So when the inspector scans the QR Code they can get the site's ID. Also the client will record the time, the inspector's position coordinates and some other corresponding information. After sign in, they can see the record interface of the system. Then they should check the machines and record the details until all the machines are checked.

Shortest Route Plan. An inspector has several sites to be inspected. He needs visited all the sites. So the problem of planning the shortest route is the typical TSP (Traveling Salesman Problem) problem. There are many algorithms of the problem [10], such as greedy algorithm, SAA (Simulate Anneal Arithmetic) algorithms, ACO (ant colony optimization) algorithm, Genetic Algorithm and so on. In this paper we use the greedy algorithm to solve the problem. Greedy algorithm, at the time of solving the problem, always makes the choice which at present appears to be the best. In other 
words, it is not the optimal while the whole is taken into account. It only has got the best only in the local optimal solution. The key of the greedy algorithm is the greedy strategy. The greedy strategy of the paper is the Nearest Neighbor Procedure. Inspectors always choose the nearest site as the next sites in this way until all the sites are visited. The route planned by the algorithm may not be the nearest way in whole, but it is the best approximation in some situations.

This paper implements the shortest route plan by introducing BaiduMap API to the system. BaiduMap API is a set of free interfaces developed by BaiduMap Service for the developers. This paper plan route in two ways: shortest and earliest to deadline. The nearest site to the inspector is the first to be visited. The nearest site to the first site is the next to one to be visited. In this way the system always find the nearest site among the unchecked sites as the next site to be checked until all the sites is visited, which is the nearest route. Arrange the sites by the deadline date and then plan the route among the earliest deadline sites in the way of greedy algorithm. Then the last site in the last shortest route is the first site to the second earliest sites. And plan the shortest route among the second earliest sites. The other sites should be done in the same manner. In that way, the route is the earliest deadline route. The route between any two sites can be gotten by the method named walkingSearch() of the class named RoutePlanSearch. Then the distance between any two site can be gotten by the method named getDistance() of the class named RouteLine. The route plan shows in Fig.3 below.

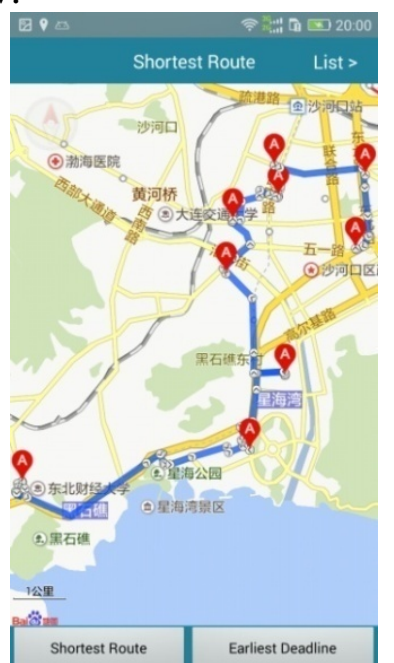

(a) Shortest Route

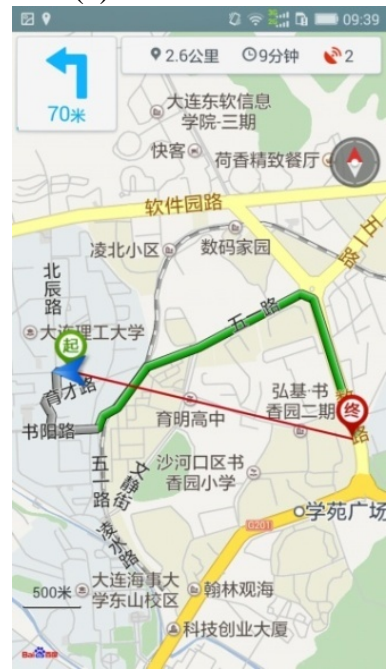

(d) Navigation

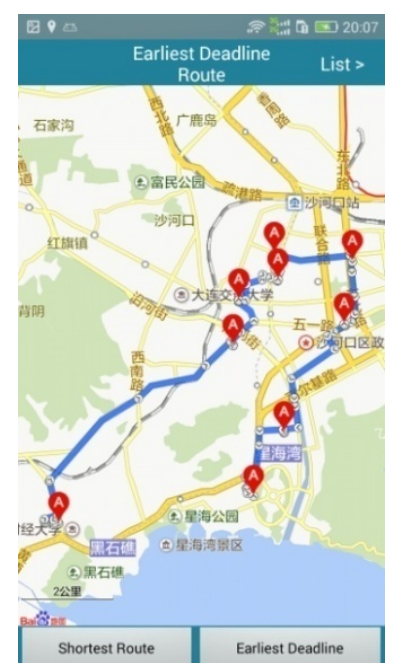

(b) Earliest Deadline Route

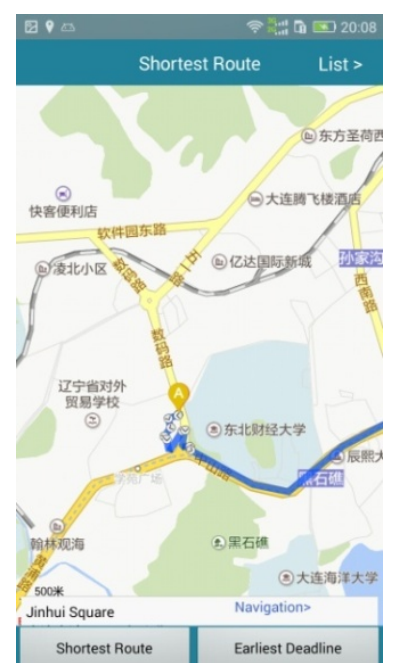

(c) View Site Detail

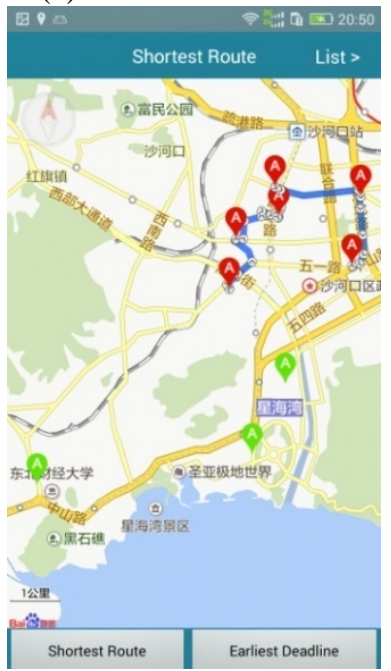

(e) Route Plan after Some Sites Are Checked

Fig.3 Route Plan 
The (a) in the Fig.3 above shows the shortest route of all the sites. The marks in the map show the sites of the inpector. The start of the route is the nearest site to the inspector's location right now. And the second site is the nearest site to the start. In this way the route can be planned out. The (b) in Fig.3 above shows the earliest deadline route. The start of the route is the nearest site to the inspector's among the earliest to deadline. In this way inspector can check the sites that will be deadline ealier than the others firstly to ensure all the site checked in the warranty period. The (c) in the Fig. 3 above shows the detail of the site. Touch the mark in the map and we can see the detail. The (d) in Fig. 3 shows the navigation of a site. Inspector can get to the site following the navigation. The (e) in Fig.3 shows the route plan of part of the sites. The green marks show the checked sites. The route is planned among the sites unchecked.

\section{Summary}

This paper has designed and implemented the lift inspection system. The system implements the function of sign in by QR Code by introducing the open source tool named ZXing. In this way the fake record is avoided. This will ease the burden of the managers. By this the work of supervision has been enhanced. With the supervision the inspectors will work more hardly and improve the efficiency of the inpection. Also the embedded database SQLite is used to store data, such as task, record, in the local-storage. In this way inspectors can record and view the task even without network and can submit the record when there is network after record. Additionally this will save the money of the inspectors, because they can use the wifi to download and upload data instead of data usage. The system also implements the function of planning route by introducing BaiduMap API to the system with greedy algorithm. This can be very useful to the inspectores. Inspectors can know the sites very well from the map. They can also plan the inspection route by themselves with the all the sites shown in the map. Finally navigation is shown to the inspectors so that inspectors can find the road to the sites to be visited easily. The navigation can help the inspectors find the sites easily. That will be a great help to those who are not familiar to the location. It will save a lot of time and improve the efficiency of inspection.

Also there are also some shortcomings. The route plan algorithm is optimal partly, the optimal in the whole is not solved. This also shows the new direction of the next work.

\section{References}

[1] Chen Wei-zhang, "The inspection system that is based on the mobile internet. " Jilin University, China, Master’s paper, 2015.

[2] Zhou pan, Zhang Lei, "An Android Platform Based Information Acquisition System for Transformer Stations”. Computer Measurement \& Control, 2015,09:3073-3076.

[3] Wang Chang-biao, "Design and Implementation of Android Application for Inspection System. ” Beijing University of Posts and Telecommunications, Master’s paper, 2014.

[4] Rong Yan-dong. "Application Research of Mabatis Persistence Layer Framework. ” Information Security and Technology, 2015,12:86-88.

[5] Ren Zhong-fang, Zhang Hua, “Overview of the Research in Model-View-Controller Pattern,” Application Research of Computers, 2004,10:1-4+8.

[6] Xue Feng, Liang Feng, "Research on Spring MVC Framework Based Web and Its Application.” Journal of Hefei University of Technology (Natural Science), 2012,03:337-340.

[7] Tang Min, Song Jie, “The Mechanism and Applications of SQLite.” Computer Knowledge and Technology, 2008,04:600-603.

[8] Wan Ma-ning, Guan Yong, "Research on Typical Technologies of Embedded Database-SQLite and Berkeley DB.” Microcomputer Information, 2006,02:91-93+272. 
[9] Lin Cun-yan,"Design and Implementation of Generation and Decoding of QR Code Based on Android Platform.” Shandong Normal University, China, Master’s paper, 2015.

[10] Denso Wave Inc., “QR Code information homepage,” http://www.qrcode.com/en/index.html.

[11] Tian Gui-chao, Li Ming, "Several Methods for Solving Traveling Salesman Problem." Computer Simulation, 2006,08:153-157. 